\title{
Discrete Spectral Transformations of Skew Orthogonal Polynomials and Associated Discrete Integrable Systems
}

\author{
Hiroshi MIKI, Hiroaki GODA and Satoshi TSUJIMOTO \\ Department of Applied Mathematics and Physics, Graduate School of Informatics, \\ Kyoto University, Sakyo-Ku, Kyoto 606 8501, Japan \\ E-mail:miki@amp.i.kyoto-u.ac.jp, tujimoto@i.kyoto-u.ac.jp \\ URL: http://www-is.amp.i.kyoto-u.ac.jp/lab/en/miki/
}

Received December 01, 2011, in final form February 20, 2012; Published online February 29, 2012 http://dx.doi.org/10.3842/SIGMA.2012.008

\begin{abstract}
Discrete spectral transformations of skew orthogonal polynomials are presented. From these spectral transformations, it is shown that the corresponding discrete integrable systems are derived both in $1+1$ dimension and in $2+1$ dimension. Especially in the $(2+1)$ dimensional case, the corresponding system can be extended to $2 \times 2$ matrix form. The factorization theorem of the Christoffel kernel for skew orthogonal polynomials in random matrix theory is presented as a by-product of these transformations.
\end{abstract}

Key words: skew orthogonal polynomials; discrete integrable systems; discrete coupled KP equation; Pfaff lattice; Christoffel-Darboux kernel

2010 Mathematics Subject Classification: 42C05; 35C05; 37K60; 15B52

\section{Introduction}

Orthogonal polynomials and their varieties have been investigated for a long time and yielded a lot of contributions to various areas including quantum physics, random matrices, wavelets and so on. Over the last twenty years, several integrable systems, both in continuous variable and in discrete variable, have been found to relate to such polynomials through their spectral transformations. One of the well-known results is the relation between ordinary orthogonal polynomials (OPs) and Toda chain (see [5, 21] for further details). For recent years, from this point of view, new types of integrable systems have been derived [23, 22] and the algebraic structures of their solutions also have been discussed in [19, 18].

In this paper, we treat the skew orthogonal polynomials (SOPs), first introduced in the theory of random matrices [7]. It is known that the continuous spectral transformation of SOPs induces the semi-discrete integrable system, known as Pfaff lattice [2,3], and its relations to the Lie algebra, geometry and matrix eigenvalue problems are discussed in [2, 14, 15] respectively. Although, the full-discrete integrable system associated with SOPs, namely the discrete counterpart of Pfaff lattice, still remains to be found and even the discrete spectral transformation of SOPs is not obtained. Recalling the application of the discrete integrable system to several areas such as eigenvalue problems and so on (e.g. [17, 26]), the discrete integrable system associated with SOPs, if obtained, can be expected to have an application or contribution to such areas. We shall derive the corresponding full-discrete integrable systems from their discrete spectral transformations.

This paper is organized as follows. In Section 2, a brief review of the relationship between OPs and the discrete time Toda equation is given and we propose the discrete spectral transformation of SOPs. From these transformations, we derive the corresponding discrete integrable system, 
which takes $(1+1)$-dimensional form. In Section 3, another discrete integrable system associated with SOPs is shown to be derived also from their discrete spectral transformations, which takes $(2+1)$-dimensional form. In Section 4 , we also show that the system obtained in Section 3 can be extended to $2 \times 2$ matrix form. In Section 5, concluding remarks are presented.

\section{Skew orthogonal polynomials}

We first briefly review a relation between the discrete Toda time equation and the discrete spectral transformation of OPs according to [21]. The discrete time Toda equation (on semiinfinite lattice)

$$
U_{n}^{t} V_{n}^{t}=U_{n-1}^{t+1} V_{n}^{t+1}, \quad U_{n}^{t}+V_{n+1}^{t}=U_{n}^{t+1}+V_{n}^{t+1}, \quad V_{0}^{t}=0,
$$

is known to admit the following discrete Lax pair:

$$
x \Phi^{t+1}=\left(\begin{array}{ccccc}
U_{0}^{t} & 1 & & & \\
& U_{1}^{t} & 1 & & \\
& & U_{2}^{t} & 1 & \\
& & & \ddots & \ddots
\end{array}\right) \Phi^{t}, \quad \Phi^{t}=\left(\begin{array}{cccc}
1 & & & \\
V_{1}^{t} & 1 & & \\
& V_{2}^{t} & 1 & \\
& & \ddots & \ddots
\end{array}\right) \Phi^{t+1},
$$

where $x$ is a spectral parameter and $\Phi^{t}=\left(\phi_{0}^{t}(x), \phi_{1}^{t}(x), \ldots\right)^{T}$ is a wave function. From (1), we obtain

$$
x \Phi^{t+1}=\left(\begin{array}{ccccc}
U_{0}^{t}+V_{1}^{t} & 1 & & & \\
U_{1}^{t} V_{1}^{t} & U_{1}^{t}+V_{2}^{t} & 1 & & \\
& U_{2}^{t} V_{2}^{t} & U_{2}^{t}+V_{3}^{t} & 1 & \\
& & \ddots & \ddots & \ddots
\end{array}\right) \Phi^{t+1},
$$

for all $t$. If we normalize $\phi_{0}^{t}(x)=1$, Favard's theorem shows that $\left\{\phi_{n}^{t}(x)\right\}_{n=0}^{\infty}$ is a sequence of monic orthogonal polynomials, i.e. there exists some linear functional $\mathcal{L}^{t}: \mathbb{R}[x] \rightarrow \mathbb{R}$ such that

$$
\mathcal{L}^{t}\left[\phi_{m}^{t}(x) \phi_{n}^{t}(x)\right]=h_{n}^{t} \delta_{m n}, \quad h_{n}^{t} \neq 0 .
$$

Therefore, one can easily find that the discrete Lax pair (1) is just the discrete spectral transformation of orthogonal polynomials known as Christoffel transformation and Geronimus transformation $[6,8]$, respectively.

Our main aim is to derive the discrete integrable systems associated with SOPs. To that end, we shall give the discrete spectral transformations of SOPs like Christoffel and Geronimus transformation. We first begin with the definition of SOPs:

Definition 1. Let $\langle\cdot \mid \cdot\rangle$ be the bilinear 2-form from $\mathbb{R}[z] \times \mathbb{R}[z]$ to $\rightarrow \mathbb{R}$ which satisfy the skew symmetric relation

$$
\langle f(z) \mid g(z)\rangle=-\langle g(z) \mid f(z)\rangle .
$$

This skew-symmetric bilinear 2-form is often called "skew-inner product". The SOPs $\left\{q_{n}(z)\right\}_{n=0}^{\infty}$ with respect to the skew-inner product $\langle\cdot \mid \cdot\rangle$ are defined as the set of polynomials satisfying the following relations:

$$
\begin{aligned}
& \left\langle q_{2 m}(z) \mid q_{2 n+1}(z)\right\rangle=r_{n} \delta_{m n}, \quad r_{n} \neq 0, \\
& \left\langle q_{2 m}(z) \mid q_{2 n}(z)\right\rangle=-\left\langle q_{2 m+1}(z) \mid q_{2 n+1}(z)\right\rangle=0,
\end{aligned}
$$

for all nonnegative integers $m, n$. 
From now on, unless specified, we assume that SOPs are monic, which ensures the uniqueness of SOPs of even degree. However, SOPs of odd degree have the following ambiguity: the skew orthogonality relation (2) is invariant under the replacement

$$
q_{2 n+1}(z) \rightarrow q_{2 n+1}(z)+\alpha_{n} q_{2 n}(z),
$$

for any $\alpha_{n} \in \mathbb{R}$. In many cases, the coefficient of $z^{2 n}$ in $q_{2 n+1}(z)$ is usually chosen as 0 and then the SOPs are uniquely determined.

The discrete spectral transformation of SOPs (that is, the transformation from SOPs to another SOPs) can be constructed.

Theorem 1. Let $\left\{q_{n}(z)\right\}_{n=0}^{\infty}$ be SOPs with respect to the skew-inner product $\langle\cdot \mid \cdot\rangle$. Let us denote some constant parameter by $\lambda$ satisfying

$$
\lambda \in\left\{z \in \mathbb{C} \mid q_{2 n}(z) \neq 0, \quad \text { for all } n=0,1, \ldots\right\} .
$$

Consider the new sequence of polynomials $\left\{q_{n}^{*}(z)\right\}_{n=0}^{\infty}$ defined by

$$
\begin{aligned}
& q_{2 n}^{*}(z)=\frac{1}{z-\lambda}\left(\sum_{k=0}^{n} \frac{r_{n}}{r_{k}} \cdot \frac{q_{2 k+1}(z) q_{2 k}(\lambda)-q_{2 k+1}(\lambda) q_{2 k}(z)}{q_{2 n}(\lambda)}\right), \\
& q_{2 n+1}^{*}(z)=\frac{1}{z-\lambda}\left(q_{2 n+2}(z)-\frac{q_{2 n+2}(\lambda)}{q_{2 n}(\lambda)} q_{2 n}(z)\right)+\alpha_{n} q_{2 n}^{*}(z),
\end{aligned}
$$

where $\alpha_{n}$ is an arbitrary parameter. Then $\left\{q_{n}^{*}(z)\right\}_{n=0}^{\infty}$ are again SOPs with respect to the modified skew-inner product

$$
\langle\cdot \mid \cdot\rangle^{*}:=\langle(z-\lambda) \cdot \mid(z-\lambda) \cdot\rangle
$$

Proof. In this proof, because of the equation (3), we take the arbitrary parameter $\alpha_{n}=0$ for all $n$ in (5) for simplicity. In order to prove the theorem, it is enough to check the skew orthogonal relation (2) for $\left\{q_{n}^{*}(z)\right\}_{n=0}^{\infty}$. Using the skew orthogonality relation (2) for $\left\{q_{n}(z)\right\}_{n=0}^{\infty}$, we find

$$
\begin{aligned}
& \left\langle q_{2 n}^{*}(z), q_{2 m+1}^{*}(z)\right\rangle^{*}=\left\langle(z-\lambda) q_{2 n}^{*}(z),(z-\lambda) q_{2 m+1}^{*}(z)\right\rangle \\
& \quad=\left\langle\sum_{k=0}^{n} \frac{q_{2 k}(\lambda) q_{2 k+1}(z)-q_{2 k+1}(\lambda) q_{2 k}(z)}{r_{k} r_{n}^{-1} q_{2 n}(\lambda)} \mid q_{2 m+2}(z)-\frac{q_{2 m+2}(\lambda)}{q_{2 m}(\lambda)} q_{2 m}(z)\right\rangle \\
& \quad=\frac{r_{n}}{q_{2 n}(\lambda)} \sum_{k=0}^{n}\left\langle\frac{q_{2 k}(\lambda) q_{2 k+1}(z)}{r_{k}} \mid q_{2 m+2}(z)-\frac{q_{2 m+2}(\lambda)}{q_{2 m}(\lambda)} q_{2 m}(z)\right\rangle=\frac{q_{2 n+2}(\lambda)}{q_{2 n}(\lambda)} r_{n} \delta_{m n} .
\end{aligned}
$$

Similarly, direct calculations also show us

$$
\left\langle q_{2 m}^{*}(z), q_{2 n}^{*}(z)\right\rangle^{*}=\left\langle q_{2 n+1}^{*}(z), q_{2 m+1}^{*}(z)\right\rangle^{*}=0 .
$$

This completes the proof.

Now observing the discrete spectral transformation of even-degree SOPs (4), one can easily find that except for the multiplier factor, this is equivalent to the Christoffel-Darboux kernel for skew orthogonal polynomials (we will call this "skew-Christoffel kernel") $[9,16]$ introduced in the theory of random matrices which takes the form

$$
I_{N}(x, y)=\sum_{k=0}^{N} \frac{q_{2 k+1}(x) q_{2 k}(y)-q_{2 k+1}(y) q_{2 k}(x)}{r_{k}},
$$

where $\left\{q_{n}(z)\right\}_{n=0}^{\infty}$ are some SOPs. We hence obtain the following corollary: 
Corollary 1. The skew-Christoffel kernel (7) can be factorized into the two SOPs:

$$
I_{N}(x, y)=(x-y) \frac{q_{2 N}(x) q_{2 N}^{*}(x)}{r_{N}},
$$

where the SOPs $q_{2 N}(x)$ and $q_{2 N}^{*}(x)$ are connected to each other by the spectral transformation (2) with $z=x, \lambda=y$.

There exist many works on the skew-Christoffel kernel for the specific weight cases (see e.g. $[1,9]$ ). Here we stress that the factorization (8) holds not only for the case mentioned above but also for any well-defined skew inner product. Especially, consider the skew inner product in orthogonal and symplectic ensemble given by:

$$
\begin{aligned}
\langle f(z) \mid g(z)\rangle_{\text {orthogonal }} & =\iint_{\mathbb{D}^{2}} \operatorname{sgn}\left(z_{1}-z_{2}\right) f\left(z_{1}\right) g\left(z_{2}\right) w\left(z_{1}\right) w\left(z_{2}\right) d z_{1} d z_{2}, \\
\langle f(z) \mid g(z)\rangle_{\text {symplectic }} & =\int_{\mathbb{D}}\left(f(z) g^{\prime}(z)-f^{\prime}(z) g(z)\right) w(z) d z,
\end{aligned}
$$

where $f^{\prime}(z)=\frac{d}{d z} f(z)$ and $\mathbb{D}, w(z)$ are the corresponding integral domain and weight function respectively. For such skew inner product, the modified skew inner product (6) can be written as follows:

$$
\begin{aligned}
& \langle(z-\lambda) f(z) \mid(z-\lambda) g(z)\rangle_{\text {orthogonal }}=\iint_{\mathbb{D}^{2}} \operatorname{sgn}\left(z_{1}-z_{2}\right) f\left(z_{1}\right) g\left(z_{2}\right) \bar{w}\left(z_{1}\right) \bar{w}\left(z_{2}\right) d z_{1} d z_{2}, \\
& \langle(z-\lambda) f(z) \mid(z-\lambda) g(z)\rangle_{\text {symplectic }}=\int_{\mathbb{D}}\left(f(z) g^{\prime}(z)-f^{\prime}(z) g(z)\right) \bar{w}(z) d z,
\end{aligned}
$$

with $\bar{w}(z)=(z-\lambda) w(z)$. This shows that the skew-Christoffel kernel in random matrix theory can be factorized into two even-degree SOPs with respect to the different weight function.

Remark 1. Similar relations hold between the Christoffel transformation of OPs and the Christoffel kernels. Hence we call the discrete spectral transformation (4), (5) as the skewChristoffel transformation.

Iterating the skew-Christoffel transformation, we obtain a sequence of SOPs by the following procedure:

$$
\begin{aligned}
& q_{n}^{0}(z):=q_{n}(z) \\
& q_{2 n}^{t+1}(z):=\frac{1}{z-\lambda}\left(q_{2 n+1}^{t}(z)+\sum_{k=0}^{n} A_{n, k}^{t} q_{2 k}^{t}(z)+\sum_{k=0}^{n-1} B_{n, k}^{t} q_{2 k+1}^{t}(z)\right) \\
& q_{2 n+1}^{t+1}(z):=\frac{1}{z-\lambda}\left(q_{2 n+2}^{t}(z)+C_{n}^{t} q_{2 n}^{t}(z)\right)+\alpha_{n}^{t} q_{2 n}^{t+1}(z)
\end{aligned}
$$

where

$$
A_{n, k}^{t}=\frac{r_{n}^{t}}{r_{k}^{t}} \cdot \frac{q_{2 k}^{t}(\lambda)}{q_{2 n}^{t}(\lambda)}, \quad B_{n, k}^{t}=-\frac{r_{n}^{t}}{r_{k}^{t}} \cdot \frac{q_{2 k+1}^{t}(\lambda)}{q_{2 n}^{t}(\lambda)}, \quad C_{n}^{t}=-\frac{q_{2 n+2}^{t}(\lambda)}{q_{2 n}^{t}(\lambda)} .
$$

Here we denote the corresponding skew-inner product by $\langle\cdot \mid \cdot\rangle^{t}$. Then, skew-orthogonality relations are given as follows:

$$
\begin{aligned}
& \langle\cdot \mid \cdot\rangle^{0}:=\langle\cdot \mid \cdot\rangle, \quad\langle\cdot \mid \cdot\rangle^{t+1}:=\langle(z-\lambda) \cdot \mid(z-\lambda) \cdot\rangle^{t}, \\
& \left\langle q_{2 m}^{t}(z), q_{2 n+1}^{t}(z)\right\rangle^{t}=r_{n}^{t} \delta_{m n}, \quad\left\langle q_{2 m}^{t}(z), q_{2 n}^{t}(z)\right\rangle^{t}=\left\langle q_{2 m+1}^{t}(z), q_{2 n+1}^{t}(z)\right\rangle^{t}=0 .
\end{aligned}
$$

For simplicity, we assume $\alpha_{n}^{t}=0$. 
We then proceed to find the inverse transformation of the skew-Christoffel transformation. Using the skew-orthogonality relation (2), we can indeed construct the different contiguous relations between $\left\{q_{n}^{t}(z)\right\}_{n=0}^{\infty}$ and $\left\{q_{n}^{t+1}(z)\right\}_{n=0}^{\infty}$ as follows:

$$
\begin{aligned}
& q_{2 n}^{t}(z)=q_{2 n}^{t+1}(z)+\sum_{k=0}^{n-1} \alpha_{n, k}^{t} q_{2 k}^{t+1}(z)+\sum_{k=0}^{n-1} \beta_{n, k}^{t} q_{2 k+1}^{t+1}(z), \\
& q_{2 n+1}^{t}(z)=q_{2 n+1}^{t+1}(z)+\sum_{k=0}^{n} \gamma_{n, k}^{t} q_{2 k}^{t+1}(z)+\sum_{k=0}^{n-1} \epsilon_{n, k}^{t} q_{2 k+1}^{t+1}(z),
\end{aligned}
$$

where

$$
\begin{aligned}
\alpha_{n, k}^{t} & =\frac{1}{r_{k}^{t+1}}\left\langle(z-\lambda) q_{2 n}^{t+1}(z) \mid(z-\lambda) q_{2 k+1}^{t+1}(z)\right\rangle^{t}, \\
\beta_{n, k}^{t} & =\frac{1}{r_{k}^{t+1}}\left\langle(z-\lambda) q_{2 k}^{t+1}(z) \mid(z-\lambda) q_{2 n}^{t+1}(z)\right\rangle^{t}, \\
\gamma_{n, k}^{t} & =\frac{1}{r_{k}^{t+1}}\left\langle(z-\lambda) q_{2 n+1}^{t}(z) \mid(z-\lambda) q_{2 k+1}^{t+1}(z)\right\rangle^{t}, \\
\epsilon_{n, k}^{t} & =\frac{1}{r_{k}^{t+1}}\left\langle(z-\lambda) q_{2 k}^{t+1}(z) \mid(z-\lambda) q_{2 n+1}^{t}(z)\right\rangle^{t} .
\end{aligned}
$$

Combining (10) and (11), we can form the following Lax pair:

$$
\begin{gathered}
(z-\lambda) \Phi^{t+1}=L^{t} \Phi^{t}:=\left(\begin{array}{ccccccc}
A_{0,0}^{t} & 1 & & & & \\
C_{0}^{t} & 0 & 1 & & & \\
A_{1,0}^{t} & B_{1,0}^{t} & A_{1,1}^{t} & 1 & & \\
0 & 0 & C_{1}^{t} & 0 & 1 & \\
\vdots & \ddots & \ddots & \ddots & \ddots & \ddots
\end{array}\right) \Phi^{t}, \\
\Phi^{t}=R^{t} \Phi^{t+1}:=\left(\begin{array}{ccccc}
1 & & & & \\
\gamma_{0,0}^{t} & 1 & & & \\
\alpha_{1,0}^{t} & \beta_{1,0}^{t} & 1 & & \\
\gamma_{1,0}^{t} & \epsilon_{1,0}^{t} & \gamma_{1,1}^{t} & 1 & \\
\vdots & \ddots & \ddots & \ddots & \ddots
\end{array}\right) \Phi^{t+1}, \\
\end{gathered}
$$

where the wave-function $\Phi^{t}=\left(q_{0}^{t}(z), q_{1}^{t}(z), q_{2}^{t}(z), \ldots\right)^{T}$ is the sequence of SOPs. From the compatibility condition of (12), the discrete Lax equation is derived:

$$
L^{t} R^{t}=R^{t+1} L^{t+1}
$$

We can observe that if we take the suitable limit, the second equation of (10) goes to the relation:

$$
\frac{d}{d t}\langle\cdot \mid \cdot\rangle=\langle z \cdot \mid \cdot\rangle+\langle\cdot \mid z \cdot\rangle,
$$

which appears in the Pfaff lattice proposed by Adler et al. [2]. The discrete Lax equation (13) thus can be considered to be the discrete counterpart of the Pfaff lattice.

\section{Discrete integrable systems of SOPs in $2+1$ dimension}

In the previous section, we derive the discrete integrable system (13) from the discrete spectral transformation of SOPs. However the system is non-local and therefore complicated. The 
similar problem occurs in the case of ordinary biorthogonal polynomials and discrete twodimensional Toda (discrete KP) equation. This problem can be solved by introducing "another time-evolution" [25]. Furthermore, observing the relation between skew-Christoffel kernel and SOPs (8), one can realize that only even-degree SOPs appear. We hence expect that evendegree SOPs play an crucial role in the corresponding discrete integrable systems. Due to the guiding principles mentioned above, we shall construct the discrete integrable systems in $(2+1)$-dimensional form associated with only even-degree SOPs.

Let $\langle\cdot \mid \cdot\rangle^{s, t}$ be a skew-inner product such that

$$
\langle\cdot \mid \cdot\rangle^{s+1, t}=\langle(z-\mu) \cdot \mid(z-\mu) \cdot\rangle^{s, t}, \quad\langle\cdot \mid \cdot\rangle^{s, t+1}=\langle(z-\lambda) \cdot \mid(z-\lambda) \cdot\rangle^{s, t},
$$

where we assume $\lambda \neq \mu$. We denote the corresponding SOPs by $\left\{q_{n}^{s, t}(z)\right\}_{n=0}^{\infty}$. From Theorem 1 , $\left\{q_{n}^{s, t}(z)\right\}_{n=0}^{\infty}$ and $\left\{q_{n}^{s+1, t}(z)\right\}_{n=0}^{\infty}$ are connected by the relation (4) and (5) with $\lambda=\mu$. Similarly, $\left\{q_{n}^{s, t}(z)\right\}_{n=0}^{\infty}$ are also related to $\left\{q_{n}^{s, t+1}(z)\right\}_{n=0}^{\infty}$ by (4) and (5).

We shall find the local contiguous relations among $\left\{q_{n}^{s, t}(z)\right\}_{n=0}^{\infty}$. To that end, we first recall the Pfaffian expression of ordinary SOPs [2], which will play a crucial role in the following sections. Pfaffians are defined by:

$$
\operatorname{Pf}\left(i_{0}, \ldots, i_{2 n-1}\right):=\sum_{\sigma \in \mathfrak{S}_{2 n}} \frac{\operatorname{sgn} \sigma}{n ! 2^{n}} \prod_{0 \leq i \leq n-1} \operatorname{Pf}\left(i_{\sigma(2 i)}, i_{\sigma(2 i+1)}\right),
$$

where the elements of $\operatorname{Pfaffians} \operatorname{Pf}(i, j)$ are supposed to satisfy the skew-symmetric relation:

$$
\operatorname{Pf}(i, j)=-\operatorname{Pf}(j, i) .
$$

From the definition of Pfaffians, we can find the following properties of Pfaffians which resemble those of determinants:

$$
\begin{aligned}
& \operatorname{Pf}\left(i_{0}, \ldots, i_{2 n-1}\right)=\sum_{k=0}^{2 n-2}(-1)^{k}\left(i_{k}, i_{2 n-1}\right)\left(i_{0}, \ldots, \widehat{i_{k}}, \ldots, i_{2 n-2}\right), \\
& \operatorname{Pf}\left(i_{0}, \ldots, a \cdot i_{a}+b \cdot i_{b}, \ldots, i_{2 n-1}\right) \\
& \quad=a \operatorname{Pf}\left(i_{0}, \ldots, i_{a}, \ldots, i_{2 n-1}\right)+b \operatorname{Pf}\left(i_{0}, \ldots, i_{b}, \ldots, i_{2 n-1}\right), \\
& \operatorname{Pf}(\ldots, s, \ldots, s, \ldots)=0,
\end{aligned}
$$

where $\widehat{j}$ means the deletion of $j$ and

$$
\operatorname{Pf}\left(i, a \cdot i_{a}+b \cdot i_{b}\right):=a \operatorname{Pf}\left(i, i_{a}\right)+b \operatorname{Pf}\left(i, i_{b}\right) .
$$

For further details of Pfaffians, see e.g. [13, 20].

Employing the "skew" moments $\left\langle z^{i} \mid z^{j}\right\rangle$ as the elements of Pfaffians, SOPs can be written in terms of Pfaffians as well as ordinary OPs in terms of determinants.

Proposition $1([2])$. The SOPs with respect to $\langle\cdot \mid \cdot\rangle$ can be expressed in terms of Pfaffians:

$$
\begin{aligned}
& q_{2 n}(z)=\frac{\operatorname{Pf}(0,1, \ldots, 2 n, z)}{\operatorname{Pf}(0,1, \ldots, 2 n-1)}, \\
& q_{2 n+1}(z)=q_{2 n}(z)=\frac{\operatorname{Pf}(0,1, \ldots, 2 n-1,2 n+1, z)}{\operatorname{Pf}(0,1, \ldots, 2 n-1)}+\alpha_{n} q_{2 n}(z),
\end{aligned}
$$

where $\alpha_{n}$ is an arbitrary parameter and the elements of Pfaffians are defined by

$$
\operatorname{Pf}(i, j)=\left\langle z^{i} \mid z^{j}\right\rangle, \quad \operatorname{Pf}(i, z)=z^{i}
$$

for all nonnegative integers $i, j$. 
Extending this proposition, we have obtained the Pfaffian expressions of the time-evolved even degree SOPs.

Theorem 2. The even degree SOPs $\left\{q_{2 n}^{s, t}(z)\right\}_{n=0}^{\infty},\left\{q_{2 n}^{s+1, t}(z)\right\}_{n=0}^{\infty},\left\{q_{2 n}^{s, t+1}(z)\right\}_{n=0}^{\infty},\left\{q_{2 n}^{s+1, t+1}(z)\right\}_{n=0}^{\infty}$ can be written in terms of the moments with respect to $\langle\cdot \mid \cdot\rangle^{s, t}$ :

$$
\begin{aligned}
& q_{2 n}^{s, t}(z)=\frac{\operatorname{Pf}(0,1, \ldots, 2 n, z)}{\operatorname{Pf}(0,1, \ldots, 2 n-1)}, \\
& q_{2 n}^{s+1, t}(z)=\frac{1}{z-\mu} \cdot \frac{\operatorname{Pf}(0,1, \ldots, 2 n+1, \mu, z)}{\operatorname{Pf}(0,1, \ldots, 2 n, \mu)}, \\
& q_{2 n}^{s, t+1}(z)=\frac{1}{z-\lambda} \cdot \frac{\operatorname{Pf}(0,1, \ldots, 2 n+1, \lambda, z)}{\operatorname{Pf}(0,1, \ldots, 2 n, \lambda)}, \\
& q_{2 n}^{s+1, t+1}(z)=\frac{1}{(z-\mu)(z-\lambda)} \cdot \frac{\operatorname{Pf}(0,1, \ldots, 2 n+2, \mu, \lambda, z)}{\operatorname{Pf}(0,1, \ldots, 2 n+1, \mu, \lambda)},
\end{aligned}
$$

where the elements of Pfaffians are defined by

$$
\begin{aligned}
& \operatorname{Pf}(i, j)=s_{i, j}^{s, t}:=\left\langle z^{i} \mid z^{j}\right\rangle^{s, t}, \\
& \operatorname{Pf}(i, z)=z^{i}, \quad \operatorname{Pf}(i, \lambda)=\lambda^{i}, \quad \operatorname{Pf}(i, \mu)=\mu^{i}, \\
& \operatorname{Pf}(z, \mu)=\operatorname{Pf}(\mu, \lambda)=\operatorname{Pf}(\lambda, z)=0 .
\end{aligned}
$$

Before proving this theorem, we show the properties of the Pfaffian elements.

Lemma 1. Employing the notation (19) in (17), for the elements of the Pfaffians defined by (21), the following properties hold:

$$
\begin{aligned}
& \operatorname{Pf}((i+1)-\mu \cdot i,(j+1)-\mu \cdot j)=\operatorname{Pf}(i+1, j+1)-\mu \operatorname{Pf}(i+1, j)-\mu \operatorname{Pf}(i, j)+\mu^{2} \operatorname{Pf}(i, j), \\
& \operatorname{Pf}((i+1)-\mu \cdot i, \mu)=0 .
\end{aligned}
$$

Proof. Following the definition (19) and anti-symmetric property of Pfaffian elements (15), one can find

$$
\begin{aligned}
\operatorname{Pf}((i+1)-\mu \cdot i,(j+1)-\mu \cdot j)=\operatorname{Pf}((i+1)-\mu \cdot i, j+1)-\mu \operatorname{Pf}((i+1)-\mu \cdot i, j) \\
\quad=-\operatorname{Pf}(j+1,(i+1)-\mu \cdot i,)+\mu \operatorname{Pf}(j, \mu(i+1)-\mu \cdot i) \\
\quad=-\operatorname{Pf}(j+1, i+1)-\mu \operatorname{Pf}(j+1, i)+\mu \operatorname{Pf}(j, i+1)-\mu^{2} \operatorname{Pf}(j, i) \\
\quad=\operatorname{Pf}(i+1, j+1)-\mu \operatorname{Pf}(i+1, j)-\mu \operatorname{Pf}(i, j)+\mu^{2} \operatorname{Pf}(i, j) .
\end{aligned}
$$

One can also find

$$
\operatorname{Pf}(i+1-\mu \cdot i, \mu)=\operatorname{Pf}(i+1, \mu)-\mu \operatorname{Pf}(i, \mu)=\mu^{i+1}-\mu^{i+1}=0 .
$$

This completes the proof.

Proof of Theorem 2. We introduce the function $\tau_{n}^{s, t}$ and $\hat{\tau}_{n}^{s, t}$ as follows:

$$
\tau_{n}^{s, t}=\operatorname{Pf}(0, \ldots, 2 n-1), \quad \hat{\tau}_{n}^{s, t}=\operatorname{Pf}(0, \ldots, 2 n, z) .
$$

It is also convenient to introduce the shift operator $T_{s}$ and $T_{t}$ satisfying

$$
T_{s}[f(s)]=f(s+1), \quad T_{t}[f(t)]=f(t+1)
$$

and the notation

$$
a_{b}:=a-b .
$$


From Proposition 1 and the relation (14), It is easy to see

$$
q_{2 n}^{s, t}(z)=\frac{\hat{\tau}_{n}^{s, t}}{\tau_{n}^{s, t}}
$$

and

$$
\begin{aligned}
& T_{s}[\operatorname{Pf}(i, j)]=\operatorname{Pf}(i+1, j+1)-\mu \operatorname{Pf}(i+1, j)-\mu \operatorname{Pf}(i, j+1)+\mu^{2} \operatorname{Pf}(i, j), \\
& T_{t}[\operatorname{Pf}(i, j)]=\operatorname{Pf}(i+1, j+1)-\lambda \operatorname{Pf}(i+1, j)-\lambda \operatorname{Pf}(i, j+1)+\lambda^{2} \operatorname{Pf}(i, j) .
\end{aligned}
$$

With the help of (17) and (18), we can see

$$
\begin{aligned}
\operatorname{Pf}(0, \ldots, 2 n, \mu) & =\operatorname{Pf}(0, \ldots, 2 n, \mu)-\mu \operatorname{Pf}(0, \ldots, 2 n-1,2 n-1, \mu) \\
& =\operatorname{Pf}(0, \ldots, 2 n-1, \widehat{2 n-1}, \mu),
\end{aligned}
$$

where $\widetilde{j}$ denotes $(j+1)-\mu \cdot j$ in the sense of (17). Iterating this transformation, we can find

$$
\operatorname{Pf}(0, \ldots, 2 n, \mu)=\operatorname{Pf}(0, \widetilde{0}, \widetilde{1}, \ldots, \widetilde{2 n-1}, \mu) .
$$

Meanwhile, we can also find

$$
\begin{aligned}
& \operatorname{Pf}\left(0, \widetilde{i_{0}}, \ldots, \widetilde{i_{2 n-1}}, \mu\right) \\
& \quad=\operatorname{Pf}\left(\widetilde{i_{0}}, \ldots, \widetilde{i_{2 n-1}}\right)-\sum_{k=0}^{2 n-1}(-1)^{k} \operatorname{Pf}\left(\widetilde{i_{k}}, \mu\right) \operatorname{Pf}\left(0, \widetilde{i_{0}}, \ldots, \widetilde{\hat{i}_{k}}, \ldots, \widetilde{i_{2 n-1}}, \mu\right),
\end{aligned}
$$

where we have used the Pfaffian expansion (16). With the help of Lemma 1 and (24), it is straightforward to see

$$
\operatorname{Pf}(\widetilde{i}, \widetilde{j})=T_{s}[\operatorname{Pf}(i, j)], \quad \operatorname{Pf}(\widetilde{i}, \mu)=0 .
$$

Therefore, combining (25) and (26), we arrive at

$$
T_{s}[\operatorname{Pf}(0, \ldots, 2 n-1)]=\operatorname{Pf}(0, \ldots, 2 n, \mu) .
$$

In a similar manner, by using $\operatorname{Pf}(\widetilde{i}, \lambda)=(\lambda-\mu) \lambda^{i}$, we further obtain

$$
\operatorname{Pf}(0, \ldots, 2 n+1, \mu, \lambda)=\lambda_{\mu} T_{s}[\operatorname{Pf}(0, \ldots, 2 n, \lambda)] .
$$

Finally, arranging these results, we have

$$
\begin{aligned}
& \tau_{n}^{s+1, t}=\operatorname{Pf}(0, \ldots, 2 n, \mu), \quad \tau_{n}^{s, t+1}=\operatorname{Pf}(0, \ldots, 2 n, \lambda), \\
& \tau_{n}^{s+1, t+1}=\lambda_{\mu}^{-1} \operatorname{Pf}(0, \ldots, 2 n+1, \mu, \lambda) .
\end{aligned}
$$

From these results, we can easily see that $\hat{\tau}_{n}^{s, t}$ is equal to $\tau_{n}^{s+1, t}$ with the substitution $z=\lambda$. Hence, in a similar manner, we can also obtain

$$
\begin{aligned}
& \hat{\tau}_{n}^{s+1, t}=z_{\mu}^{-1} \operatorname{Pf}(0, \ldots, 2 n+1, \mu, z), \quad \hat{\tau}_{n}^{s, t+1}=z_{\lambda}^{-1} \operatorname{Pf}(0, \ldots, 2 n+1, \lambda, z), \\
& \hat{\tau}_{n}^{s+1, t+1}=z_{\mu}^{-1} z_{\lambda}^{-1} \lambda_{\mu}^{-1} \operatorname{Pf}(0, \ldots, 2 n+2, \mu, \lambda, z) .
\end{aligned}
$$

Finally, from (23), (27) and (28), we obtain the relation (20). This completes the proof.

Using Theorem 2, we can obtain the following contiguous relations of even-degree SOPs. 
Theorem 3. The series of even degree SOPs $\left\{q_{2 n}^{s, t}(z)\right\}_{n=0}^{\infty}$ satisfy the following contiguous relations:

$$
\begin{aligned}
& z_{\lambda} q_{2 n}^{s, t+1}(z)-z_{\mu} q_{2 n}^{s+1, t}(z)=z_{\mu, \lambda} A_{n}^{s, t} q_{2 n-2}^{s+1, t+1}(z)-B_{n}^{s, t} q_{2 n}^{s, t}(z), \\
& z_{\mu, \lambda} q_{2 n}^{s+1, t+1}(z)-q_{2 n+2}(z)=z_{\lambda} C_{n}^{s, t} q_{2 n}^{s, t+1}(z)-z_{\mu} D_{n}^{s, t} q_{2 n}^{s+1, t},
\end{aligned}
$$

with

$$
\begin{array}{rlrl}
A_{n}^{s, t} & =(\mu-\lambda) \frac{\tau_{n+1}^{s, t} \tau_{n-1}^{s+1, t+1}}{\tau_{n}^{s+1, t} \tau_{n}^{s, t+1}}, & B_{n}^{s, t} & =(\mu-\lambda) \frac{\tau_{n}^{s, t} \tau_{n}^{s+1, t+1}}{\tau_{n}^{s+1, t} \tau_{n}^{s, t+1}}, \\
C_{n}^{s, t} & =(\mu-\lambda)^{-1} \frac{\tau_{n+1}^{s+1, t} \tau_{n}^{s, t+1}}{\tau_{n+1}^{s, t} \tau_{n}^{s+1, t+1}}, & D_{n}^{s, t}=(\mu-\lambda)^{-1} \frac{\tau_{n+1}^{s, t+1} \tau_{n}^{s+1, t}}{\tau_{n+1}^{s, t} \tau_{n}^{s+1, t+1}},
\end{array}
$$

where $\tau_{n}^{s, t}$ is defined in (22).

Proof. Using the identities of Pfaffians [13]:

$$
\begin{aligned}
& \operatorname{Pf}\left(i_{0}, \ldots, i_{2 n-1}, a, b, c, d\right) \operatorname{Pf}\left(i_{0}, \ldots, i_{2 n-1}\right)=\operatorname{Pf}\left(i_{0}, \ldots, i_{2 n-1}, a, b\right) \operatorname{Pf}\left(i_{0}, \ldots, i_{2 n-1}, c, d\right) \\
& \quad-\operatorname{Pf}\left(i_{0}, \ldots, i_{2 n-1}, a, c\right) \operatorname{Pf}\left(i_{0}, \ldots, i_{2 n-1}, b, d\right) \\
& \quad+\operatorname{Pf}\left(i_{0}, \ldots, i_{2 n-1}, a, d\right) \operatorname{Pf}\left(i_{0}, \ldots, i_{2 n-1}, b, c\right)
\end{aligned}
$$

and

$$
\begin{aligned}
& \operatorname{Pf}\left(i_{0}, \ldots, i_{2 n}, a, b, c\right) \operatorname{Pf}\left(i_{0}, \ldots, i_{2 n}, d\right)=\operatorname{Pf}\left(i_{0}, \ldots, i_{2 n}, a, b, d\right) \operatorname{Pf}\left(i_{0}, \ldots, i_{2 n}, c\right) \\
& \quad-\operatorname{Pf}\left(i_{0}, \ldots, i_{2 n}, a, c, d\right) \operatorname{Pf}\left(i_{0}, \ldots, i_{2 n}, b\right) \\
& \quad+\operatorname{Pf}\left(i_{0}, \ldots, i_{2 n}, b, c, d\right) \operatorname{Pf}\left(i_{0}, \ldots, i_{2 n}, a\right)
\end{aligned}
$$

we obtain the relation between $\tau_{n}^{s, t}$ and $\hat{\tau}_{n}^{s, t}$ :

$$
\begin{aligned}
& z_{\mu, \lambda} \lambda_{\mu} \tau_{n+1}^{s, t} \hat{\tau}_{n}^{s+1, t+1}=z_{\lambda} \tau_{n+1}^{s+1, t} \hat{\tau}_{n}^{s, t+1}-z_{\mu} \tau_{n+1}^{s, t+1} \hat{\tau}_{n}^{s+1, t}+\lambda_{\mu} \hat{\tau}_{n+1}^{s, t} \tau_{n}^{s+1, t+1}, \\
& \lambda_{\mu} \tau_{n}^{s+1, t+1} \hat{\tau}_{n}^{s, t}=z_{\mu} \tau_{n}^{s, t+1} \hat{\tau}_{n}^{s+1, t}-z_{\lambda} \tau_{n}^{s+1, t} \hat{\tau}_{n}^{s, t+1}+z_{\mu, \lambda} \tau_{n+1}^{s, t} \hat{\tau}_{n-1}^{s+1, t+1},
\end{aligned}
$$

where $z_{\mu, \lambda}=z_{\mu} z_{\lambda}$. Hence, from Theorem 2, we can easily arrive at the result.

Observing Theorem 3, we can regard the relation (29) as the discrete Lax pair whose wave function is the sequence of even-degree SOPs. Then we can directly obtain the following system.

Theorem 4. From the compatibility condition of (29), we can derive the following nonlinear difference system:

$$
\begin{aligned}
& A_{n}^{s+1, t+1}-A_{n+1}^{s, t}+B_{n+1}^{s, t}-B_{n}^{s+1, t+1}=C_{n}^{s, t+1}-C_{n}^{s+1, t}+D_{n}^{s+1, t}-D_{n}^{s, t+1}, \\
& A_{n}^{s+1, t} C_{n-1}^{s+1, t}=A_{n}^{s, t} C_{n}^{s, t}, \quad A_{n}^{s, t+1} D_{n-1}^{s, t+1}=A_{n}^{s, t} D_{n}^{s, t} \\
& B_{n}^{s+1, t} D_{n}^{s+1, t}=B_{n+1}^{s, t} D_{n}^{s, t}, \quad B_{n}^{s, t+1} C_{n}^{s, t+1}=B_{n+1}^{s, t} C_{n}^{s, t} .
\end{aligned}
$$

This system has a special solution which can be written in terms of Pfaffians as (30).

We can consider the system (34) as a discrete integrable system since this admits a discrete Lax pair by construction and we can put plenty arbitrary parameters into the solution (30).

From the point of view of the $\tau$-function $\tau_{n}^{s, t}$, the relation (24) which the elements of the Pfaffian satisfy is the same as the dispersion relation of the discrete coupled KP (dcKP) equation [10] and indeed one can notice that the equation (33) is just the same as the dcKP equation. Under the choice of $z$ as the spectral parameter, the dcKP equation yields the discrete Lax pair (29). Therefore the system (34) can be regarded as the nonlinear form of the dcKP. 


\section{An extension to $2 \times 2$ matrix form}

In the previous section, we propose the discrete integrable system in (2+1)-dimensional form (34) from the discrete spectral transformation of the SOPs. This system, by construction, is related only to the even-degree SOPs and not to odd-degree SOPs. Taking it into account that the integrable systems associated with both even and odd degree SOPs were already derived [1, 27], we can expect that there should exist a discrete integrable system in $2+1$ dimension which is also connected to the discrete spectral transformations of both even and odd degree SOPs. In order to construct such system, as is described in the previous section, the ambiguity of odd-degree SOPs (3) is the barrier. Conversely, in this section, we shall construct such system not avoiding the ambiguity of odd-degree SOPs but utilizing this.

Using the skew-inner product $\langle\cdot \mid \cdot\rangle^{s, t}$ with the property (14), we introduce the new sequence of polynomials $\left\{\phi_{n}^{s, t}(z)\right\}_{n=0}^{\infty}$ :

$$
\phi_{2 n}^{s, t}(z)=\frac{{\hat{\tau_{n}}}^{s, t}}{{\sigma_{n}^{s, t}}^{s,}}, \quad \phi_{2 n+1}^{s, t}(z)=\frac{\hat{\sigma}_{n}^{s, t}}{\tau_{n}^{s, t}},
$$

where $\sigma_{n}^{s, t}$ and $\hat{\sigma}_{n}^{s, t}$ are defined by

$$
\begin{aligned}
& \sigma_{n}^{s, t}:=\operatorname{Pf}(0, \ldots, 2 n-2,2 n)+(s \mu+t \lambda) \tau_{n}^{s, t}, \\
& \hat{\sigma}_{n}^{s, t}:=\operatorname{Pf}(0, \ldots, 2 n-1,2 n+1, z)+(s \mu+t \lambda) \hat{\tau}_{n}^{s, t},
\end{aligned}
$$

respectively. Recall that $\tau_{n}^{s, t}$ and $\hat{\tau}_{n}^{s, t}$ are defined by (22). here we remark that $\phi_{2 n}^{s, t}(z)$ are no longer monic except for $s=t=0$. Nevertheless, from Proposition 1, one can easily find $\left\{\phi_{n}^{s, t}(z)\right\}_{n=0}$ are also SOPs with respect to $\langle\cdot \mid \cdot\rangle^{s, t}$.

As is seen in the previous section, in order to derive the discrete Lax pair of even-degree SOPs, that is the relation between $\tau_{n}^{s, t}$ and $\hat{\tau}_{n}^{s, t}$, the equation (33) plays a central role. According to this observation, we wish to acquire the relation between $\tau_{n}^{s, t}, \hat{\tau}_{n}^{s, t}, \sigma_{n}^{s, t}$ and $\hat{\sigma}_{n}^{s, t}$. First we examine the properties of $\sigma_{n}^{s, t}$ and $\hat{\sigma}_{n}^{s, t}$.

Lemma 2. The function $\sigma_{n}^{s, t}$ and $\hat{\sigma}_{n}^{s, t}$ defined in (36) hold the following properties:

$$
\begin{aligned}
& \sigma_{n}^{s+1, t}=\operatorname{Pf}(0, \ldots, 2 n-1,2 n+1, \mu)+(s \mu+t \lambda) \tau_{n}^{s+1, t}, \\
& \sigma_{n}^{s, t+1}=\operatorname{Pf}(0, \ldots, 2 n-1,2 n+1, \lambda)+(s \mu+t \lambda) \tau_{n}^{s, t+1}, \\
& \sigma_{n}^{s+1, t+1}=\lambda_{\mu}^{-1} \operatorname{Pf}(0, \ldots, 2 n, 2 n+2, \mu, \lambda)+(s \mu+t \lambda) \tau_{n}^{s+1, t+1}, \\
& \hat{\sigma}_{n}^{s+1, t}=z_{\mu}^{-1} \operatorname{Pf}(0, \ldots, 2 n, 2 n+2, \mu, z)+(s \mu+t \lambda) \hat{\tau}_{n}^{s+1, t}, \\
& \hat{\sigma}_{n}^{s, t+1}=z_{\lambda}^{-1} \operatorname{Pf}(0, \ldots, 2 n, 2 n+2, \lambda, z)+(s \mu+t \lambda) \hat{\tau}_{n}^{s, t+1}, \\
& \hat{\sigma}_{n}^{s+1, t+1}=\lambda_{\mu}^{-1} z_{\mu, \lambda}^{-1} \operatorname{Pf}(0, \ldots, 2 n+1,2 n+3, \mu, \lambda, z)+(s \mu+t \lambda) \hat{\tau}_{n}^{s+1, t+1} .
\end{aligned}
$$

Proof. In a similar manner to the proof of Theorem 2, we can see

$$
\begin{aligned}
& \operatorname{Pf}(0, \ldots, \widehat{k+1}, \ldots, 2 n+1, \mu)=\operatorname{Pf}(0, \ldots, k, k+2, \widetilde{k+2}, \ldots, \widetilde{2 n}, \mu) \\
&=\operatorname{Pf}(0, \ldots, k, k+2, \widehat{k+2}, \ldots, \widetilde{2 n}, \mu)-\mu^{2} \operatorname{Pf}(0, \ldots, k, k, \widetilde{k+2}, \ldots, \widetilde{2 n}, \mu) \\
&=\operatorname{Pf}\left(0, \ldots k,(k+2)-\mu^{2} \cdot k, \widetilde{k+2}, \ldots, \widetilde{2 n}, \mu\right) \\
&=\operatorname{Pf}\left(0, \widetilde{0}, \ldots \widetilde{k},(k+2)-\mu^{2} \cdot k, \widetilde{k+2}, \ldots, \widetilde{2 n}, \mu\right) .
\end{aligned}
$$

Noticing $(k+2)-\mu^{2} \cdot k=\widetilde{k+1}-\mu \cdot \widetilde{k}$, we get

$$
\operatorname{Pf}(0, \ldots, \widehat{k+1}, \ldots, 2 n+1, \mu)=\operatorname{Pf}(0, \widetilde{0}, \ldots, \widehat{\widetilde{k}}, \ldots, \widetilde{2 n}, \mu)-\mu \operatorname{Pf}(0, \widetilde{0}, \ldots, \widehat{\widehat{k+1}}, \ldots, \widetilde{2 n}, \mu)
$$




$$
=T_{s}[\operatorname{Pf}(0, \ldots, \widehat{k}, \ldots, 2 n-1)]-\mu T_{s}[\operatorname{Pf}(0, \ldots, \widehat{k+1}, \ldots, 2 n-1)],
$$

where we have used the relation (26). Therefore with the symmetry $\mu \leftrightarrow \lambda$, we have

$$
\begin{aligned}
& T_{s}[\operatorname{Pf}(0,1, \ldots, 2 n-2,2 n)]=\operatorname{Pf}(0,1, \ldots, 2 n-1,2 n+1, \mu)-\mu \tau_{n}^{s+1, t}, \\
& T_{t}[\operatorname{Pf}(0,1, \ldots, 2 n-2,2 n)]=\operatorname{Pf}(0,1, \ldots, 2 n-1,2 n+1, \mu)-\lambda \tau_{n}^{s, t+1} .
\end{aligned}
$$

In a similar manner, we can also obtain

$$
\begin{aligned}
& T_{s} \circ T_{t}[\operatorname{Pf}(0,1, \ldots, 2 n-2,2 n)]=\lambda_{\mu}^{-1} \operatorname{Pf}(0,1, \ldots, 2 n-1,2 n+1, \mu)-(\mu+\lambda) \tau_{n}^{s+1, t+1}, \\
& T_{s}[\operatorname{Pf}(0,1, \ldots, 2 n-1,2 n+1, z)]=z_{\mu}^{-1} \operatorname{Pf}(0,1, \ldots, 2 n, 2 n+2, \mu, z)-\mu \hat{\tau}_{n}^{s+1, t}, \\
& T_{t}[\operatorname{Pf}(0,1, \ldots, 2 n-1,2 n+1, z)]=z_{\lambda}^{-1} \operatorname{Pf}(0,1, \ldots, 2 n, 2 n+2, \lambda, z)-\lambda \hat{\tau}_{n}^{s, t+1}, \\
& T_{s} \circ T_{t}[\operatorname{Pf}(0,1, \ldots, 2 n-1,2 n+1, z)] \\
& \quad=z_{\mu, \lambda}^{-1} \operatorname{Pf}(0,1, \ldots, 2 n+1,2 n+3, \mu, \lambda, z)-(\mu+\lambda) \hat{\tau}_{n}^{s+1, t+1} .
\end{aligned}
$$

These results immediately lead us to the conclusion.

By virtue of Lemma 2, we can find the relation between $\tau_{n}^{s, t}, \hat{\tau}_{n}^{s, t}, \sigma_{n}^{s, t}$ and $\hat{\sigma}_{n}^{s, t}$.

Theorem 5. The function $\tau_{n}^{s, t}, \hat{\tau}_{n}^{s, t}, \sigma_{n}^{s, t}$ and $\hat{\sigma}_{n}^{s, t}$ defined in (22) and (36) satisfy the following four relations:

$$
\begin{aligned}
& z_{\mu, \lambda} \lambda_{\mu} \sigma_{n+1}^{s, t} \hat{\tau}_{n-1}^{s+1, t+1}=z_{\lambda} \tau_{n}^{s+1, t} \hat{\sigma}_{n}^{s, t+1}-z_{\mu} \tau_{n}^{s, t+1} \hat{\sigma}_{n}^{s+1, t}+\lambda_{\mu} \sigma_{n}^{s+1, t+1} \hat{\tau}_{n}^{s, t}, \\
& z_{\mu, \lambda} \lambda_{\mu} \tau_{n+1}^{s, t} \hat{\sigma}_{n-1}^{s+1, t+1}=z_{\lambda} \sigma_{n}^{s+1, t} \hat{\tau}_{n}^{s, t+1}-z_{\mu} \sigma_{n}^{s, t+1} \hat{\tau}_{n}^{s+1, t}+\lambda_{\mu} \tau_{n}^{s+1, t+1} \hat{\sigma}_{n}^{s, t}, \\
& z_{\mu, \lambda} \lambda_{\mu} \sigma_{n+1}^{s, t} \hat{\tau}_{n}^{s+1, t+1}=z_{\lambda} \tau_{n+1}^{s+1, t} \hat{\sigma}_{n}^{s, t+1}-z_{\mu} \tau_{n+1}^{s, t+1} \hat{\sigma}_{n}^{s+1, t}+\lambda_{\mu} \sigma_{n}^{s+1, t+1} \hat{\tau}_{n+1}^{s, t}, \\
& z_{\mu, \lambda} \lambda_{\mu} \tau_{n+1}^{s, t} \hat{\sigma}_{n}^{s+1, t+1}=z_{\lambda} \sigma_{n+1}^{s+1, t} \hat{\tau}_{n}^{s, t+1}-z_{\mu} \sigma_{n+1}^{s, t+1} \hat{\tau}_{n}^{s+1, t}+\lambda_{\mu} \tau_{n}^{s+1, t+1} \hat{\sigma}_{n+1}^{s, t} .
\end{aligned}
$$

Proof. Pfaffian identities (31), (32) and Lemma 2 directly lead us to the (37). This completes the proof.

If we take $\sigma=\tau$, one finds that the equations (37) go to the discrete coupled KP equation (33). Therefore, these equations can be considered as the natural extension of the discrete coupled $\mathrm{KP}$ equation from the point of view of the SOPs. Hereafter we will call (33) as the extended discrete coupled KP (edcKP) equation.

The edcKP equation gives us the contiguous relation among $\left\{\phi_{n}^{s, t}(z)\right\}$ :

Theorem 6. Let $\Phi_{n}^{s, t}(z)$ be the vector function given by

$$
\Phi_{n}^{s, t}(z)=\left(\begin{array}{c}
\phi_{2 n}^{s, t}(z) \\
\phi_{2 n+1}^{s, t}(z)
\end{array}\right)
$$

The vector functions $\left\{\Phi_{n}^{s, t}(z)\right\}_{n=0}^{\infty}$ satisfy the following two vector linear equations:

$$
\begin{aligned}
& z_{\lambda} \Phi_{n}^{s, t+1}(z)-z_{\mu} \Phi_{n}^{s+1, t}(z)=z_{\mu, \lambda} \mathbf{A}_{n}^{s, t} \Phi_{n-1}^{s+1, t+1}(z)-\mathbf{B}_{n}^{s, t} \Phi_{n}^{s, t}(z), \\
& z_{\mu, \lambda} \Phi_{n}^{s+1, t+1}(z)-\Phi_{n+1}(z)=z_{\lambda} \mathbf{C}_{n}^{s, t} \Phi_{n}^{s, t+1}(z)-z_{\mu} \mathbf{D}_{n}^{s, t} \Phi_{n}^{s+1, t},
\end{aligned}
$$

where the coefficients $\mathbf{A}_{n}^{s, t}, \mathbf{B}_{n}^{s, t}, \mathbf{C}_{n}^{s, t}, \mathbf{D}_{n}^{s, t}$ can be written explicitly in terms of Pfaffians:

$$
\mathbf{A}_{n}^{s, t}=(\lambda-\mu)\left(\begin{array}{cc}
0 & \frac{\tau_{n+1}^{s, t} \tau_{n-1}^{s+1, t+1}}{\sigma_{n}^{s+1, t} \sigma_{n}^{s, t+1}} \\
\frac{\sigma_{n+1}^{s, t} \sigma_{n-1}^{s+1, t+1}}{\tau_{n}^{s+1, t} \tau_{n}^{s, t+1}} & 0
\end{array}\right),
$$




$$
\begin{aligned}
& \mathbf{B}_{n}^{s, t}=(\lambda-\mu)\left(\begin{array}{cc}
0 & \frac{\tau_{n}^{s, t} \tau_{n}^{s+1, t+1}}{\sigma_{n}^{s+1, t} \sigma_{n}^{s, t+1}} \\
\frac{\sigma_{n}^{s, t} \sigma_{n}^{s+1, t+1}}{\tau_{n}^{s+1, t} \tau_{n}^{s, t+1}} & 0
\end{array}\right), \\
& \mathbf{C}_{n}^{s, t}=(\lambda-\mu)^{-1}\left(\begin{array}{cc}
0 & \frac{\tau_{n+1}^{s+1, t} \tau_{n}^{s, t+1}}{\sigma_{n+1}^{s, t} \sigma_{n}^{s+1, t+1}} \\
\frac{\sigma_{n+1}^{s+1, t} \sigma_{n}^{s, t+1}}{\tau_{n+1}^{s, t} \tau_{n}^{s+1, t+1}} & 0
\end{array}\right), \\
& \mathbf{D}_{n}^{s, t}=(\lambda-\mu)^{-1}\left(\begin{array}{cc}
0 & \frac{\tau_{n+1}^{s, t+1} \tau_{n}^{s+1, t}}{\sigma_{n+1}^{s, t} \sigma_{n}^{s+1, t+1}} \\
\frac{\sigma_{n+1}^{s, t+1} \sigma_{n}^{s+1, t}}{\tau_{n+1}^{s, t} \tau_{n}^{s+1, t+1}} & 0
\end{array}\right) .
\end{aligned}
$$

Proof. The definitions of $\left\{\phi_{n}^{s, t}\right\}_{n=0}^{\infty}$ (35) and the edcKP equation (37) directly lead us to the conclusion. This completes the proof.

We can regard (38) as the discrete vector Lax pair of SOPs of both even and odd degree. Therefore the discrete Lax pair shows us the following systems associated with SOPs of both even and odd degree, which can be regarded as a discrete integrable system along with (34):

Theorem 7. From the compatibility condition of the discrete vector Lax pair (38), we can derive the nonlinear system in $2+1$ dimension which takes the $2 \times 2$ matrix form:

$$
\begin{aligned}
& \mathbf{A}_{n}^{s+1, t+1}-\mathbf{A}_{n+1}^{s, t}+\mathbf{B}_{n+1}^{s, t}-\mathbf{B}_{n}^{s+1, t+1}=\mathbf{C}_{n}^{s, t+1}-\mathbf{C}_{n}^{s+1, t}+\mathbf{D}_{n}^{s+1, t}-\mathbf{D}_{n}^{s, t+1}, \\
& \mathbf{A}_{n}^{s+1, t} \mathbf{C}_{n-1}^{s+1, t}=\mathbf{A}_{n}^{s, t} \mathbf{C}_{n}^{s+1, t}, \quad \mathbf{A}_{n}^{s, t+1} \mathbf{D}_{n-1}^{s, t+1}=\mathbf{A}_{n}^{s, t} \mathbf{D}_{n}^{s, t}, \\
& \mathbf{B}_{n}^{s+1, t} \mathbf{D}_{n}^{s+1, t}=\mathbf{B}_{n+1}^{s, t} \mathbf{D}_{n}^{s, t}, \quad \mathbf{B}_{n}^{s, t+1} \mathbf{D}_{n}^{s, t+1}=\mathbf{B}_{n+1}^{s, t} \mathbf{D}_{n}^{s, t} .
\end{aligned}
$$

Special solutions of the nonlinear system (40) can be written in terms of Pfaffians given by (39).

One can easily find that this system is the $2 \times 2$ matrix extension of the system (40) and this is the nonlinear form of the edcKP equation. This system is clearly connected to the SOPs of both even and odd degree and from its construction, we can regard the system (40) as the discrete coutnerpart of the Pfaff lattice in $(2+1)$-dimensional form.

\section{Concluding remarks}

In this paper, we proposed the discrete spectral transformation of SOPs. From the discrete spectral transformation of SOPs, we derived the discrete integrable systems both in $(1+1)$ dimensional form and in $(2+1)$-dimensional form. In $(1+1)$-dimensional case, the system gives the integrable discretization of the Pfaff lattice, though the system is of course not local. In $(2+1)$-dimensional case, the system is local and corresponds only to the even-degree SOPs. This system is also shown to be connected to discrete coupled KP equation. The discrete coupled $\mathrm{KP}$ equation is known to be equivalent to the discretized Hirota-Ohta equation [11] in the bilinear form levels. Recently, the discrete integrable system is proposed from the auxiliary linear problems for the Hirota-Ohta (or DKP) system [4, 24].

We further extended the discrete integrable system associated with even-degree SOPs to the system associated with both even and odd degree SOPs. This system can be described in $2 \times 2$ matrix form, which is related to an extension of the discrete coupled KP equation. This 
extension has never been considered before, although it is quite natural from the aspects of SOPs.

Finally, let us mention for the relationship between our results and the random matrix theory. As a by-product of the discrete transformation of SOPs, we found the compact factorization of the kernel function of the correlation function for the orthogonal or symplectic ensembles in terms of the two even-degree SOPs with respect to the different weights. This factorization is not known as far as the authors know and indeed might be a new one. Moreover, it is known that a matrix integral over orthogonal and symplectic ensembles of Hermitian matrices can be considered as a $\tau$-function of the coupled KP hierarchy [12], while our discrete integrable systems (34) and (40) are directly connected to discrete coupled KP and its extension, respectively. We hope these results will bring a deeper understanding of the random matrix theory and several applications in its works in the near future.

\section{Acknowledgement}

The authors would like to thank the Centre de recherches mathématiques (CRM) for its hospitality. The work of H.M. is partially supported by a Grant-in-Aid for Japan Society for the Promotion of Science (JSPS) Fellows. The research of S.T. is supported in part by KAKENHI (22540224). The authors should like to thank the referees for the careful reading of the manuscript and a lot of helpful suggestions and comments.

\section{References}

[1] Adler M., Forrester P.J., Nagao T., van Moerbeke P., Classical skew orthogonal polynomials and random matrices, J. Statist. Phys. 99 (2000), 141-170, solv-int/9907001.

[2] Adler M., Horozov E., van Moerbeke P., The Pfaff lattice and skew-orthogonal polynomials, Int. Math. Res. Not. 1999 (1999), 569-588, solv-int/9903005.

[3] Adler M., van Moerbeke P., Toda versus Pfaff lattice and related polynomials, Duke Math. J. 112 (2002), $1-58$.

[4] Adler V.E., Postnikov V.V., Linear problems and Bäcklund transformations for the Hirota-Ohta system, Phys. Lett. A 375 (2011), 468-473, arXiv:1007.4698.

[5] Aptekarev A.I., Branquinho A., Marcellán F., Toda-type differential equations for the recurrence coefficients of orthogonal polynomials and Freud transformation, J. Comput. Appl. Math. 78 (1997), 139-160.

[6] Chihara T.S., An introduction to orthogonal polynomials, Mathematics and its Applications, Vol. 13, Gordon and Breach Science Publishers, New York, 1978.

[7] Dyson F.J., A class of matrix ensembles, J. Math. Phys. 13 (1972), 90-97.

[8] Geronimus J., On polynomials orthogonal with regard to a given sequence of numbers, Comm. Inst. Sci. Math. Méc. Univ. Kharkoff (4) 17 (1940), 3-18.

[9] Ghosh S., Generalized Christoffel-Darboux formula for classical skew-orthogonal polynomials, J. Phys. A: Math. Theor. 41 (2008), 435204, 29 pages, arXiv:0711.4432.

[10] Gilson C.R., Nimmo J.J.C., Tsujimoto S., Pfaffianization of the discrete KP equation, J. Phys. A: Math. Gen. 34 (2001), 10569-10575.

[11] Hirota R., Ohta Y., Hierarchies of coupled soliton equations. I, J. Phys. Soc. Japan 60 (1991), 798-809.

[12] Kakei S., Orthogonal and symplectic matrix integrals and coupled KP hierarchy, J. Phys. Soc. Japan 68 (1999), 2875-2877, solv-int/9909023.

[13] Knuth D.E., Overlapping Pfaffians, Electron. J. Combin. 3 (1996), no. 2, R5, 13 pages, math.CO/9503234.

[14] Kodama Y., Pierce V.U., Geometry of the Pfaff lattices, Int. Math. Res. Not. 2007 (2007), no. 23, Art. ID rnm120, 55 pages, arXiv:0705.0510.

[15] Kodama Y., Pierce V.U., The Pfaff lattice on symplectic matrices, J. Phys. A: Math. Theor. 43 (2010), 055206, 22 pages, arXiv:0802.2288. 
[16] Mehta M.L., Random matrices, 3rd ed., Pure and Applied Mathematics (Amsterdam), Vol. 142, Elsevier/Academic Press, Amsterdam, 2004.

[17] Minesaki Y., Nakamura Y., The discrete relativistic Toda molecule equation and a Padé approximation algorithm, Numer. Algorithms 27 (2001), 219-235.

[18] Mukaihira A., A $\tau$-function on the inhomogeneous lattice and the classical isotropic Heisenberg spin chain, J. Phys. A: Math. Theor. 41 (2008), 475201, 23 pages.

[19] Mukaihira A., Tsujimoto S., Determinant structure of non-autonomous Toda-type integrable systems, J. Phys. A: Math. Gen. 39 (2006), 779-788.

[20] Ohta Y., Special solutions of discrete integrable systems, in Discrete Integrable Systems, Lecture Notes in Phys., Vol. 644, Springer, Berlin, 2004, 57-83.

[21] Spiridonov V., Zhedanov A., Discrete Darboux transformations, the discrete-time Toda lattice, and the Askey-Wilson polynomials, Methods Appl. Anal. 2 (1995), 369-398.

[22] Spiridonov V., Zhedanov A., Spectral transformation chains and some new biorthogonal rational functions, Comm. Math. Phys. 210 (2000), 49-83.

[23] Spiridonov V.P., Tsujimoto S., Zhedanov A.S., Integrable discrete time chains for the FrobeniusStickelberger-Thiele polynomials, Comm. Math. Phys. 272 (2007), 139-165.

[24] Takasaki K., Auxiliary linear problem, difference Fay identities and dispersionless limit of Pfaff-Toda hierarchy, SIGMA 5 (2009), 109, 34 pages, arXiv:0908.3569.

[25] Tsujimoto S., Kondo K., Molecule solutions to discrete equations and orthogonal polynomials, Sūrikaisekikenkyūsho Kōkyūroku (2000), no. 1170, 1-8.

[26] Tsujimoto S., Nakamura Y., Iwasaki M., The discrete Lotka-Volterra system computes singular values, Inverse Problems 17 (2001), 53-58.

[27] van de Leur J., Matrix integrals and the geometry of spinors, J. Nonlinear Math. Phys. 8 (2001), 288-310, solv-int/9909028. 